\title{
Laboratory Evaluation of Fan-filter Units' Aerodynamic and Energy Performance
}

\author{
Tengfang Xu, Lawrence Berkeley National Laboratory \\ Ming-Shan Jeng, Industrial Technology Research Institute
}

\section{Biography}

Dr. Tengfang $\mathrm{Xu}$ is a Project Manager with the Environmental Energy Technologies Division, Lawrence Berkeley National Laboratory (LBNL), Berkeley, CA. He obtained his BS and MS degrees from Tsinghua University, Beijing, and a Ph.D. from the University of California, Berkeley. He is a licensed mechanical engineer in California with sixteen years of research and consulting experience in indoor environment, energy, human factors, and building systems. He serves as a Technical Editor for the Journal of the IEST, IL, USA, and is an Editorial Advisory Board Member for Cleanroom World, China.

Dr. Ming-Shan Jeng is a senior researcher from the Ventilation and Indoor Air Quality (IAQ) Laboratory of the Energy \& Resources Laboratories in the Industrial Technology Research Institute (ITRI) in Taiwan. He has more than 10 years of experience in ventilation system design, testing of fans and ventilation equipment, as well as noise control of HVAC systems. Dr. Jeng is currently involved in several research topics, including the development of high efficiency fanfilter units, the development of low-noise cross flow fans and a study on cleanroom airflow. He also leads a pioneer project on the investigation of thermoelectric and thermo-acoustic cooling technology.

\begin{abstract}
The paper discusses the benefits of having a consistent testing method to characterize aerodynamic and energy performance of FFUs. It presents evaluation methods of laboratorymeasured performance of ten relatively new, $1220 \mathrm{~mm}$ x $610 \mathrm{~mm}$ ( or $4 \mathrm{ft} \times 2 \mathrm{ft}$ ) fan-filter units (FFUs), and includes results of a set of relevant metrics such as energy performance indices (EPI) based upon the sample FFUs tested. This paper concludes that there are variations in FFUs' performance, and that using a consistent testing and evaluation method can generate compatible and comparable FFU performance information. The paper also suggests that benefits and opportunities exist for our method of testing FFU energy performance to be integrated in future recommended practices.
\end{abstract}

\section{Keywords}

Cleanroom, fan-filter unit (FFU), airflow, pressure, efficiency, energy performance index (EPI)

\section{Introduction}

Cleanroom HVAC systems account for a large portion of energy use in cleanrooms. Recent studies have found that the performance of HVAC systems varies significantly from cleanroom to cleanroom largely because of various contributing factors, such as contamination control 
requirements, air handling unit designs, system resistance, and efficiency levels offered by system components ${ }^{[1],[2],[3]}$. Those studies also uncovered energy-saving opportunities in cleanroom applications. Optimizing aerodynamic performance in air recirculation systems appears to be one of useful approaches to improve cleanroom energy efficiency.

Driven by the needs for specific contamination control and ease of installation and adaptability in cleanroom construction, air recirculation systems adopting fan-filter units (FFUs) are increasingly gaining popularity worldwide in recent years. An FFU is a self-contained unit normally attached to cleanroom T-bar ceilings and is used to supply filtered airflow. The FFU maintains contamination control by providing certain air change rates for a specified space. An FFU usually consists of a small fan, a controller, and a HEPA or ULPA filter enclosed in a box, which fits into common cleanroom ceiling grids (e.g., $1220 \mathrm{~mm}$ x $610 \mathrm{~mm}$ or $4 \mathrm{ft} \times 2 \mathrm{ft}$ ). The small fans force air through the filters and for an entire cleanroom. The large number of small fans can consume considerable energy in providing air recirculation.

Although FFUs are becoming more popular, their aerodynamic and energy performance can vary significantly. Where FFU applications are desired, it is useful for designers and building owners to have comparable information on FFU energy performance. This will allow selection of efficient units, thereby improving energy efficiency, while maintaining or improving contamination control. In practice, typical manufacturers data sheets usually contain numbers that are seemingly similar; they however do not mention test methods, if at all exist. Furthermore, statements of performance data that include power, airflow, and sound are commonly vague and could be misleading. For example, an FFU label containing specification such as "100 Watts, $50 \mathrm{dBA}, 90 \mathrm{fpm}$ (or $0.45 \mathrm{~m} / \mathrm{s}$ )" is ambiguous because it does not tell what "90 fpm (or $0.45 \mathrm{~m} / \mathrm{s}$ )" really refers to or the condition under which it was obtained. The likely variations of its interpretation, such as an airflow speed at the room cross-section, across the net filter media, at the face of the filter, or at an unspecified distance from the filter exit, would indicate very different performance. In practice to date, there has been no published testing method that FFU suppliers could consistently adopt when providing performance information. As a result, suppliers' data information cannot be meaningfully compared or its usefulness is minimal. To provide data for both users' and manufacturers' reference, ITRI has conducted relevant measurements to provide airflow, energy, and sound vibration performance data for FFUs on the market since $1999^{[4]}$. Part of its database was released in 2001, along with a brief description of the test procedure, which is consistent with industry standards for testing fan systems ${ }^{[5][6]}$.

This paper builds upon the new laboratory method of testing FFU energy and aerodynamic performance being developed by $\mathrm{LBNL}^{[7]}$, and evaluates the data that was newly generated by ITRI's recent work in this area. The compatible laboratory data used in this paper was based upon ten sample FFUs that entered the market since 2001. The sample FFUs presented in this paper were obtained by ITRI and tested at ITRI's laboratory facility.

\section{Objectives}

The objectives of this paper are to 1) present laboratory testing results on aerodynamic and energy performance of ten FFUs (1220 mm x $610 \mathrm{~mm}$, or $4 \mathrm{ft} \times 2 \mathrm{ft}) ; 2$ ) compare unit performance of the sample FFUs in the market; and 3) discuss benefits of a new testing 
procedure and potential opportunities of its integration into relevant recommended practice for FFU performance testing that is currently developed by IEST. This paper does not intend to evaluate the following performance metrics although they constitute important aspects of overall FFU performance: acoustic, vibration, filter efficiency, motor efficiency, filter media for controlling airborne molecular contamination, outlet flow uniformity, and in-situ performance. Some of these issues are and may be addressed in the broader context of the IEST Recommended Practices. For example, the issues on airflow uniformity are addressed in other standards, certification documents, or recommended practices ${ }^{[8],[9],[10]}$.

\section{Approaches}

The main approach is to use a test method being developed at Lawrence Berkeley National Laboratory $^{[7]}$, in collaboration with ITRI and other industrial partners, to characterize FFU aerodynamic and energy performance in laboratory setting. The major focus of this paper is to evaluate the laboratory measurement results from ten sample FFUs that were tested by ITRI from 2001 to 2003.

In this study, the FFUs were connected with an inlet chamber setup that is consistent with other standard test methods to determine a fan's aerodynamic performance ${ }^{[5]}$. The chamber contains a multiple-nozzle bank for recording airflow rates through the tested unit. The air from the mediate downstream of the FFUs was discharged to the atmosphere. A booster fan and a damper were installed at the chamber inlet to modulate air pressures inside the chamber so that the airflow across the FFUs was also controlled. Figure 1 shows the basic measurement layout.

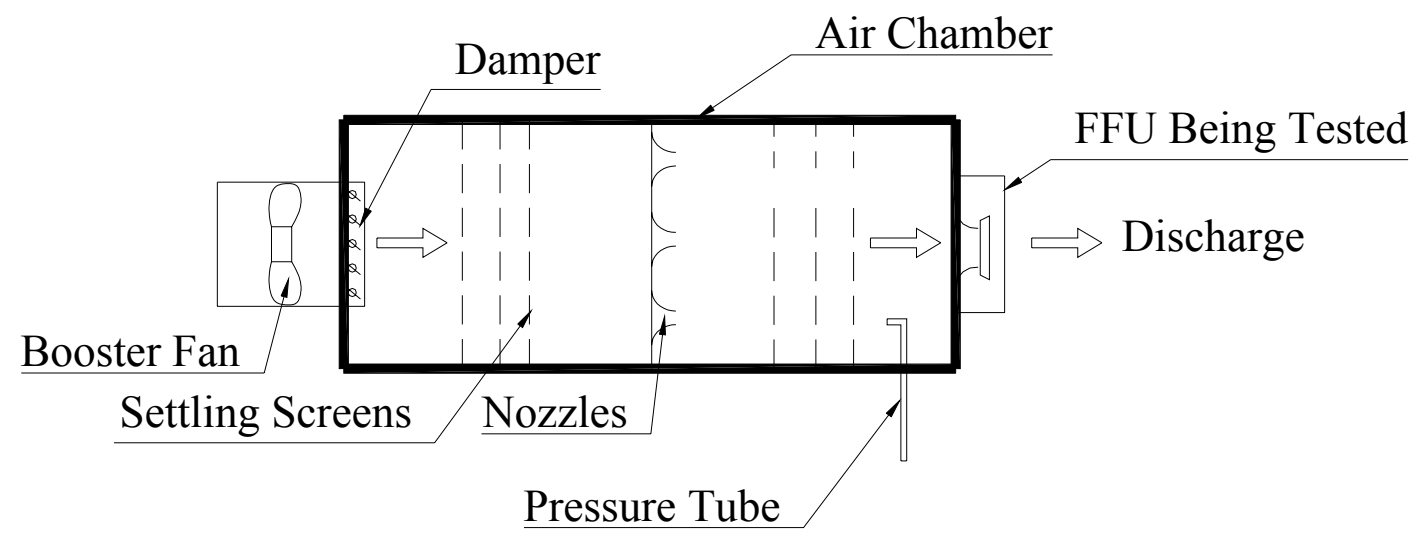

Figure 1 Laboratory measurement layout

The FFUs tested were mounted vertically on the exit end of the air chamber. Exit airflow of the FFU discharged into a room with the air at atmospheric conditions. The HEPA or ULPA filter was considered an integral part of each FFU in this survey and was not disassembled from the unit during the test. The FFU pressure rise therefore represented the pressure available after the 
filter to re-circulate the air in the cleanroom. The FFUs were tested at nearly constant rotational speeds, while static pressures at the inlet of each FFU were modulated by adjusting damper positions. Some of the FFUs were equipped with speed modulation device. When there was speed modulation device, the fan motor in FFU was set at the highest speed for testing. All of the tests were conducted during different days with somewhat different ambient conditions. The ambient conditions and the airflow conditions were recorded and were used for the air density conversion to the equivalent standard condition (i.e., $1 \mathrm{~atm}, 20^{\circ} \mathrm{C}$ ). We assume that the airflow was isothermal, although a small fraction of heat is generated from fan motors that would be transferred to the airflow. The data reported in this paper is based upon the standard air condition, i.e., with the air density of $1.20 \mathrm{~kg} / \mathrm{m}^{3}$, in order to directly compare the energy and aerodynamic performance of all FFUs.

\section{Laboratory Measurements and Metrics}

To understand the performance of each FFU unit, relevant metrics were developed to evaluate energy and aerodynamic performance for the sample units. Data analysis was then performed to quantify metrics at various conditions, and median values of energy performance were identified. The metrics, when applied uniformly, will allow direct comparison of energy performance based on manufacturers specifications or measured data. For example, this paper assesses the energy performance of ten sample FFUs using the electricity power input normalized by the airflow rate through FFUs under certain operating pressures. The metrics can be used by designers or owners in their life-cycle-cost analysis when desired. They can also be used to formulate energy-efficiency criteria for use in electric utility incentive programs. The following defines key metrics used in this paper:

- Unit Airflow Rate: Airflow rate through the FFU tested under a specific static pressure

- Airflow Speed: Unit airflow rate divided by the net FFU face area under a specific static pressure

- Total Power Usage: Total electricity power input to operate the FFU at certain airflow conditions, including the fan motor, controller, etc.

- Airflow Dynamic Power: Portion of the total power that is converted to move air through the FFU at certain airflow conditions

- Total Pressure Efficiency: Ratio of airflow dynamic power to total power input to an FFU

- Energy Performance Index (EPI): Unit's total power usage normalized by the airflow rate through the FFU under certain conditions

The uncertainty in the airflow and pressure measurements is within $\pm 2.5 \%$. A power meter measured actual power input of a FFU with the measurement uncertainty within $\pm 0.5 \%$. The total pressure is the sum of the static pressure and the dynamic pressure at a certain location of the airflow path. The total pressure rise across the FFU is measured within the chamber using the FFU exit location as the base (Figure 1). It represents the "pressure gain" as the air flowing through the unit, where the fan impeller exerts energy on the airflow. With the magnitudes of airflow speeds in the order of $0.50 \mathrm{~m} / \mathrm{s}$ (or about $100 \mathrm{fpm}$ ) or less, dynamic pressures of airflows through FFUs are usually less than $0.2 \mathrm{~Pa}$. If we consider that cleanroom system pressure drop 
(e.g., FFU systems) is around 50 to $100 \mathrm{~Pa}$ and that static pressure could be higher than $100 \mathrm{~Pa}$, dynamic pressures of airflows with speeds of $0.50 \mathrm{~m} / \mathrm{s}$ or lower would only account for an insignificant fraction $(<0.5 \%)$ of the total pressures. In this regard, values of static pressures or total pressures are expressed interchangeably in the rest of this paper.

\section{Results}

\section{General Description of the Fan-Filter Units}

This paper evaluates the energy and aerodynamic performance of ten FFUs that were originally tested by ITRI in calibrated laboratory setting. The sample FFUs were made by various major suppliers from Asia, Europe, and the US and were in use after 2001. Each of the FFUs tested has backward inclined centrifugal impellers, some of which have airfoil cross-section blades. A majority of the samples used single-phase or three-phase AC power supply, with only one powered by DC.

\section{FFU Airflow Dynamics: Airflow Rates, Pressure, and Total Pressure Efficiency}

Figure 2 shows the curves of FFU pressure rise vs. airflow speed at FFU exit. For a typical cleanroom system resistance of $100 \mathrm{~Pa}$ (or about 0.4 inch water), most of the FFUs would operate at airflow speeds typically ranging from 0.30 to $0.50 \mathrm{~m} / \mathrm{s}$ (or about 60 to $100 \mathrm{fpm}$ ). 


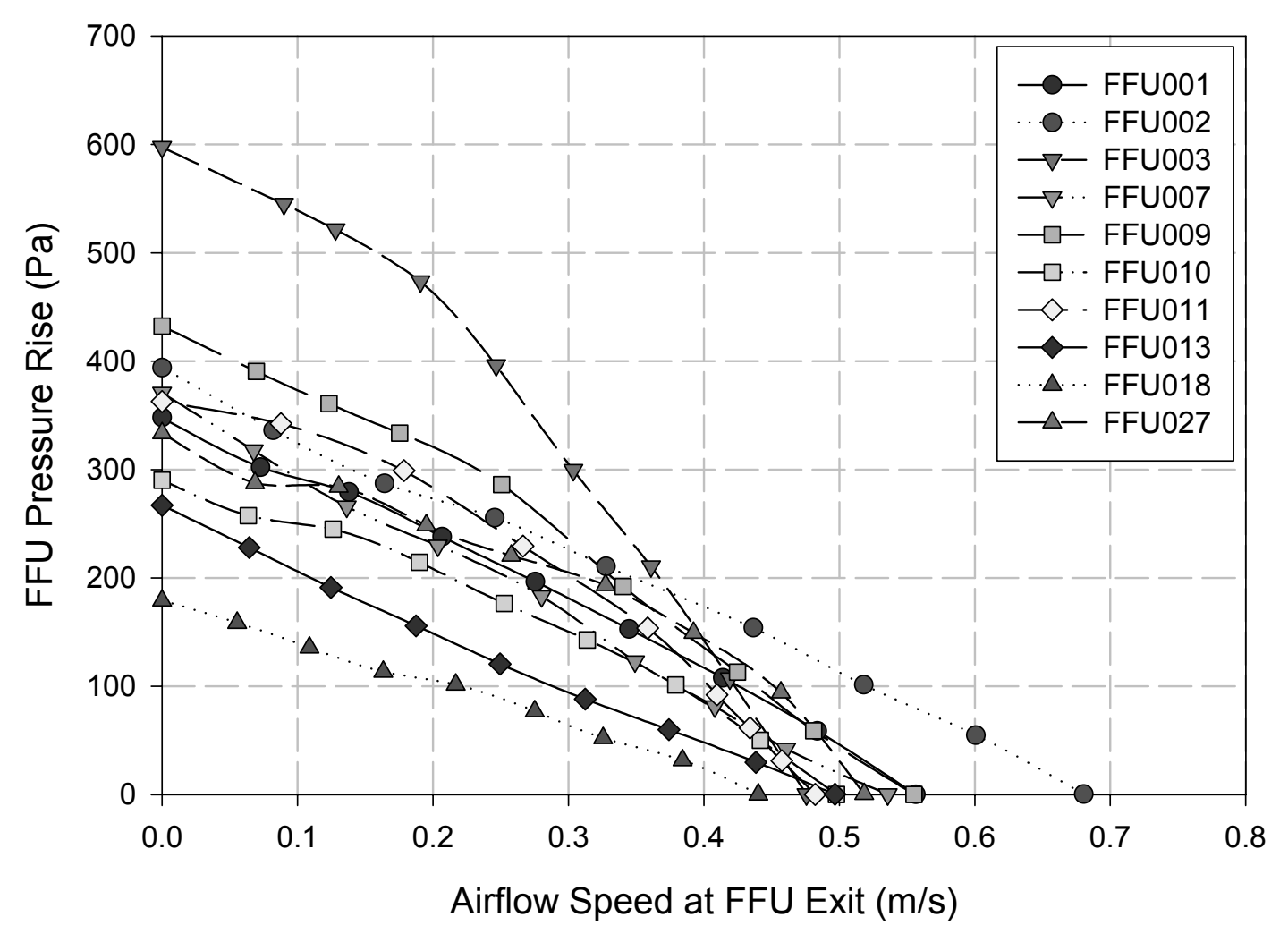

Figure 2 FFU pressure rise vs. airflow speed at FFU exit

The total FFU power efficiency $\left(E_{t}\right)$ is defined as the actual airflow dynamic power divided by the total electric power input to the FFU unit. The total FFU power efficiency includes electrical efficiency and mechanical efficiency of the whole FFU unit and it takes into account fan motors, transformers, etc. This paper does not intend to further analyze impacts from different factors associated with fan type, FFU assembly, motor type, motor control and efficiency, although these factors should be studied in future research.

$E_{t}=P_{t} Q / W$

Equation [1]

where

$\mathrm{P}_{\mathrm{t}}$ is the FFU pressure rise $(\mathrm{Pa})$

$\mathrm{Q}$ the airflow rate $\left(\mathrm{m}^{3} / \mathrm{s}\right)$, and

$\mathrm{W}$ is the total electric power input to FFU (W).

Figure 3 shows performance curves of individual FFUs in terms of their total pressure efficiency as a function of airflow speeds at the FFU exit. Total pressure efficiency of the FFUs varied considerably from unit to unit. For example, at a pressure rise of $100 \mathrm{~Pa}$ (or about 0.4 inch water), FFU airflow speeds operated in the range between 0.22 and $0.53 \mathrm{~m} / \mathrm{s}$ (or about 45 to 105 fpm), while total pressure efficiency varied approximately between $10 \%$ and $20 \%$, with a median total pressure efficiency value above $15 \%$. 
The majority of the units tested in this study produced airflow speeds within the range of 0.30 and $0.50 \mathrm{~m} / \mathrm{s}$, which is typical in cleanroom applications, at a static pressure of about $100 \mathrm{~Pa}$ (or about 0.4 inch water). In an earlier study ${ }^{[4]}$, only about half of the 20 sample units (in use before 2001) could produce airflow speeds within the range of 0.30 and $0.50 \mathrm{~m} / \mathrm{s}$ at the same static pressure of $100 \mathrm{~Pa}$ (or about 0.4 inch water). Furthermore, the median total pressure efficiency of those units was less than $14 \%$. Compared with the results from the earlier study, Figure 2 and Figure 3 indicate improvement in the aerodynamic performance of these ten FFUs over their previous counterparts. The trend of improvement might be due to a combination of factors such as technology improvement of individual FFU components, improvement in the assembly of FFUs, and other means that are inductive to design enhancement for the units. By examining the magnitudes of total pressure efficiency in this study, we can see that the efficiency of one unit could be two-to-three-times as much as others at a typical test condition. The optimal total pressure efficiency of a whole unit was normally under $20 \%$, which was much lower than that of a single fan under free flow conditions.

Based upon the above analysis, it is clear that there are considerable variations in the FFUs' aerodynamic performance from product to product. It is also clear that there is a potential for some of FFU suppliers to improve FFU aerodynamic performance under certain operating and design conditions. 


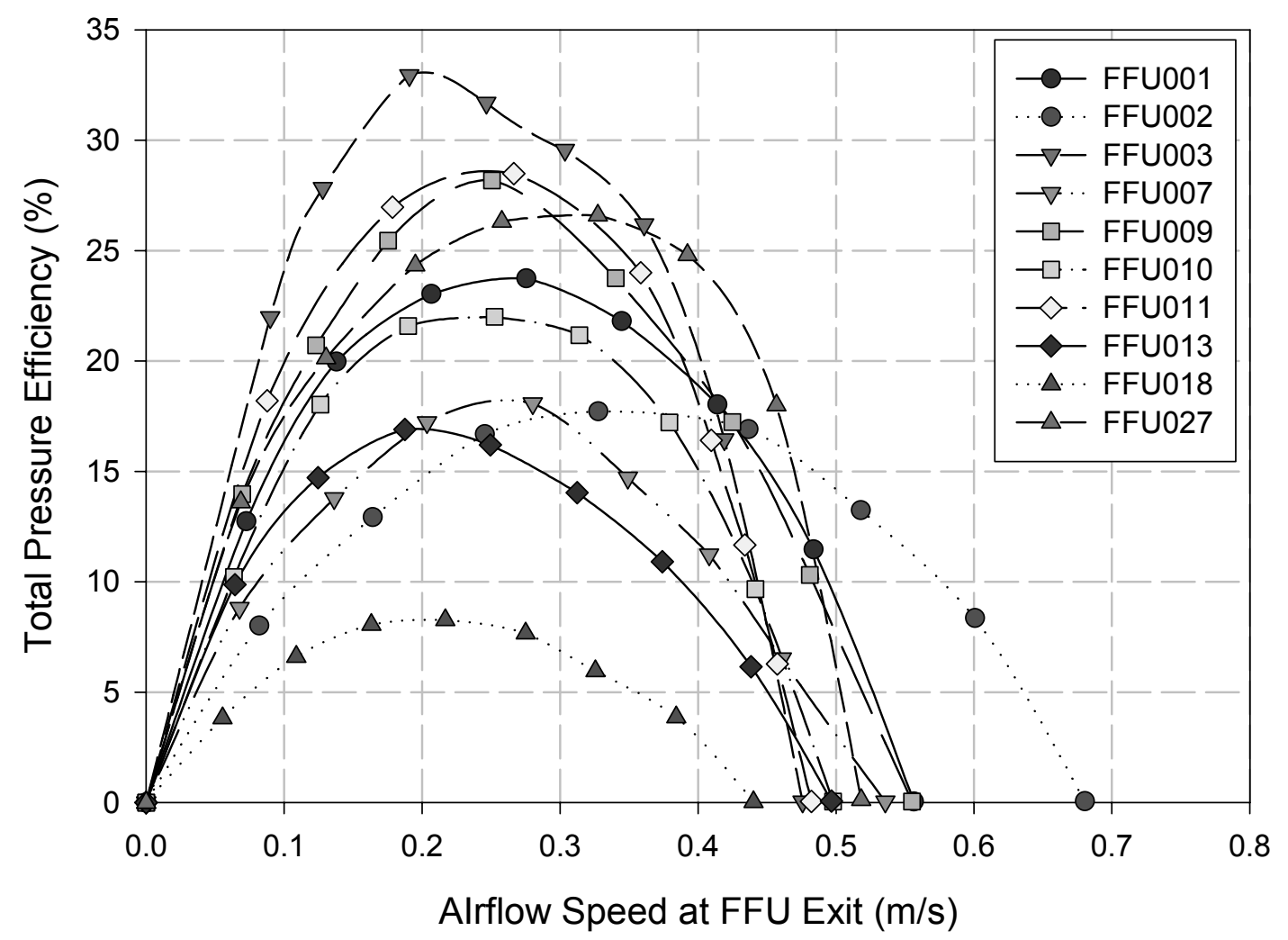

Figure 3 Total pressure efficiency vs. airflow speed at FFU exit

\section{FFU Energy Performance}

In order to evaluate the energy performance of FFUs, it is necessary to specify certain test conditions and to examine the performance metrics collectively. The following presents 1) relationships among major energy metrics and testing conditions based upon an exemplar FFU tested; 2) Energy Performance Index (EPI) corresponding to certain testing conditions based upon the all FFUs tested.

\subsection{An Exemplar Case}

With a stable rotational speed, less system-resistance associated with wider damper opening would result in higher airflows, which normally require more fan power for airflow delivery through the unit. Figure 4 shows the trend of measured air pressures (total and static), total unit efficiency, and EPI (FFU power intensity) under a range of the airflow speeds at unit's exit for an exemplar FFU.

The figure indicates that an increase in airflow speeds corresponds consistently with the decrease in static pressure (and total pressure), which was a result of wider opening of the damper coupling with the original fan features. At the static pressure of $125 \mathrm{~Pa}$ (or about 0.5 inch water), the corresponding airflow speed was about $0.39 \mathrm{~m} / \mathrm{s}$ (or about $80 \mathrm{fpm}$ ). This corresponded to FFU power intensity of about $10.6 \mathrm{~W} \mathrm{per} \mathrm{m}^{3} / \mathrm{min}$ ( or $0.30 \mathrm{~W} / \mathrm{cfm}$ ), and total pressure efficiency of nearly $20 \%$. A higher static pressure than $125 \mathrm{~Pa}$ at the inlet of the FFU corresponded with a 
lower airflow rate and higher FFU power intensity, while the total pressure efficiency can be either higher or lower depending on the actual magnitude of the airflow rates.

It is clear that in this case there was a peak in the total pressure efficiency as the airflow speed changes progressively from a low rate (e.g., $0.10 \mathrm{~m} / \mathrm{s}$ ) to a higher rate (e.g., $0.50 \mathrm{~m} / \mathrm{s}$ ) while the power intensity decreases consistently with the increase in airflow speeds. On the other hand, this indicates that the fan inside the FFU with wider damper opening would not have to work as hard as it would have to, compared to other cases in which the system-resistance increases (e.g., a narrower damper opening). In this regard, the FFU tends to be more efficient in delivering airflow at a higher airflow speed. Similar trends were also found for other FFUs tested in this dataset.

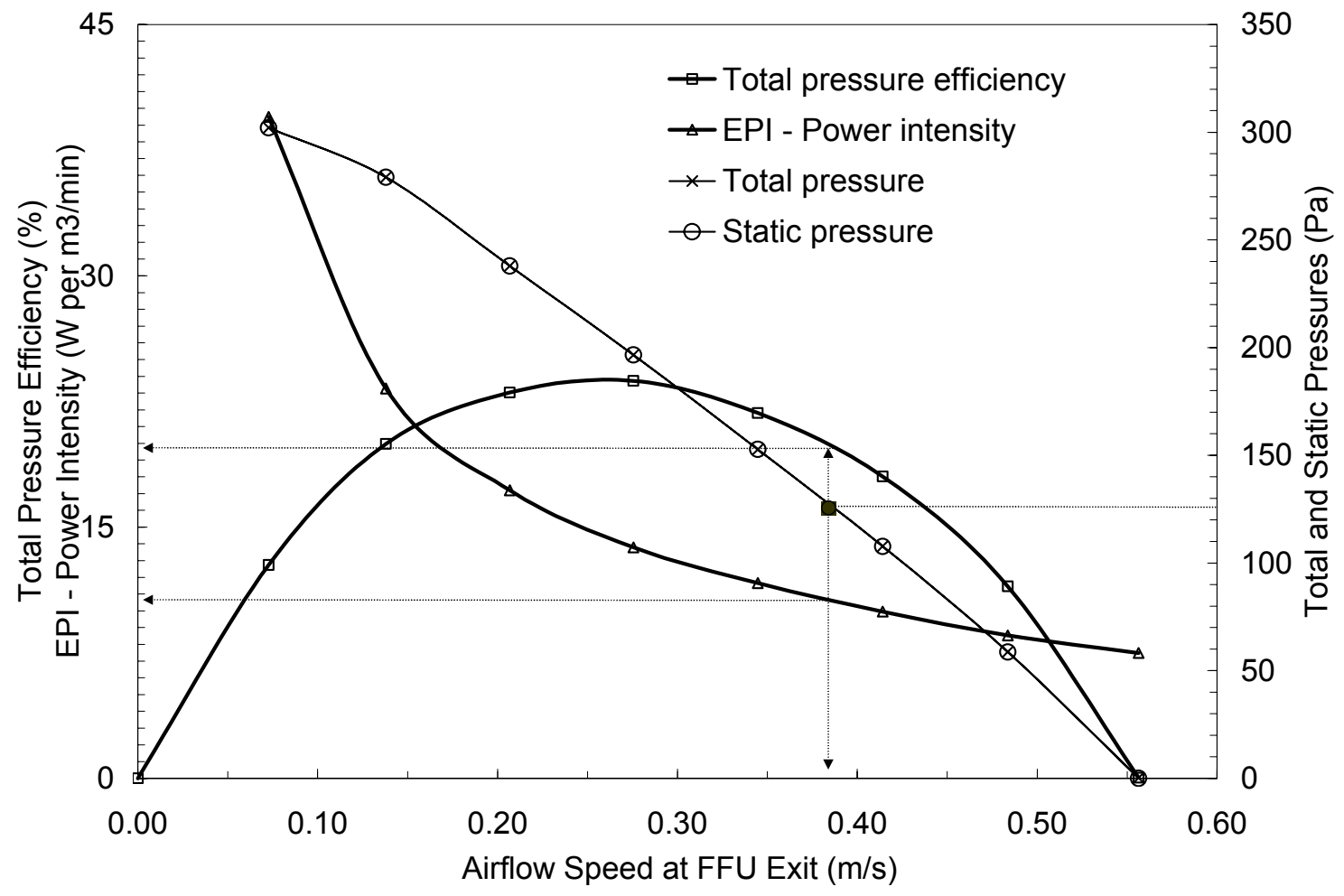

Figure 4 FFU pressures, power intensity, and total pressure efficiency

\subsection{FFU Energy Performance Index (EPI)}

In order to compare EPI values of various FFUs, it is essential to specify conditions under which these FFUs are tested. Based upon the trends illustrated in the previous section, it is clear that energy performance of an FFU was consistently associated with the static pressure at the inlet of the tested FFU. At the same static pressure condition, it is possible that actual airflow rates are different among various FFUs; therefore, the calculated EPI value of an FFU would be likely based upon an airflow rate different from others. The advantage of fixing the static pressure at 
certain level (e.g., $125 \mathrm{~Pa}$ ) is to allow a direct EPI comparison regardless differences in air systems in which FFUs would actually be installed.

Figure 5 shows the EPI values, namely electricity power intensity (watts per $\mathrm{m}^{3} / \mathrm{min}$, or W/cfm), of the ten FFUs at an inlet static pressure of $125 \mathrm{~Pa}$ (or about 0.5 inch water). The median value

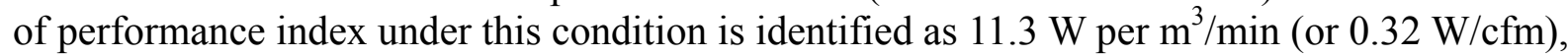
meaning that $50 \%$ of the FFUs tested at the inlet static pressure of $125 \mathrm{~Pa}$ (or about 0.5 inch water) perform better than $11.3 \mathrm{~W}$ per $\mathrm{m}^{3} / \mathrm{min}$ (or $0.32 \mathrm{~W} / \mathrm{cfm}$ ).

Overall, the differences among the unit's EPI values can be three times as much under the certain operating condition. This indicates that there is potential for many of the FFU suppliers to improve FFU energy performance. It also indicates that there is an opportunity for users to select more efficient units as a means of improving the performance of their cleanroom systems, once such comparable performance data, EPI, can be obtained.

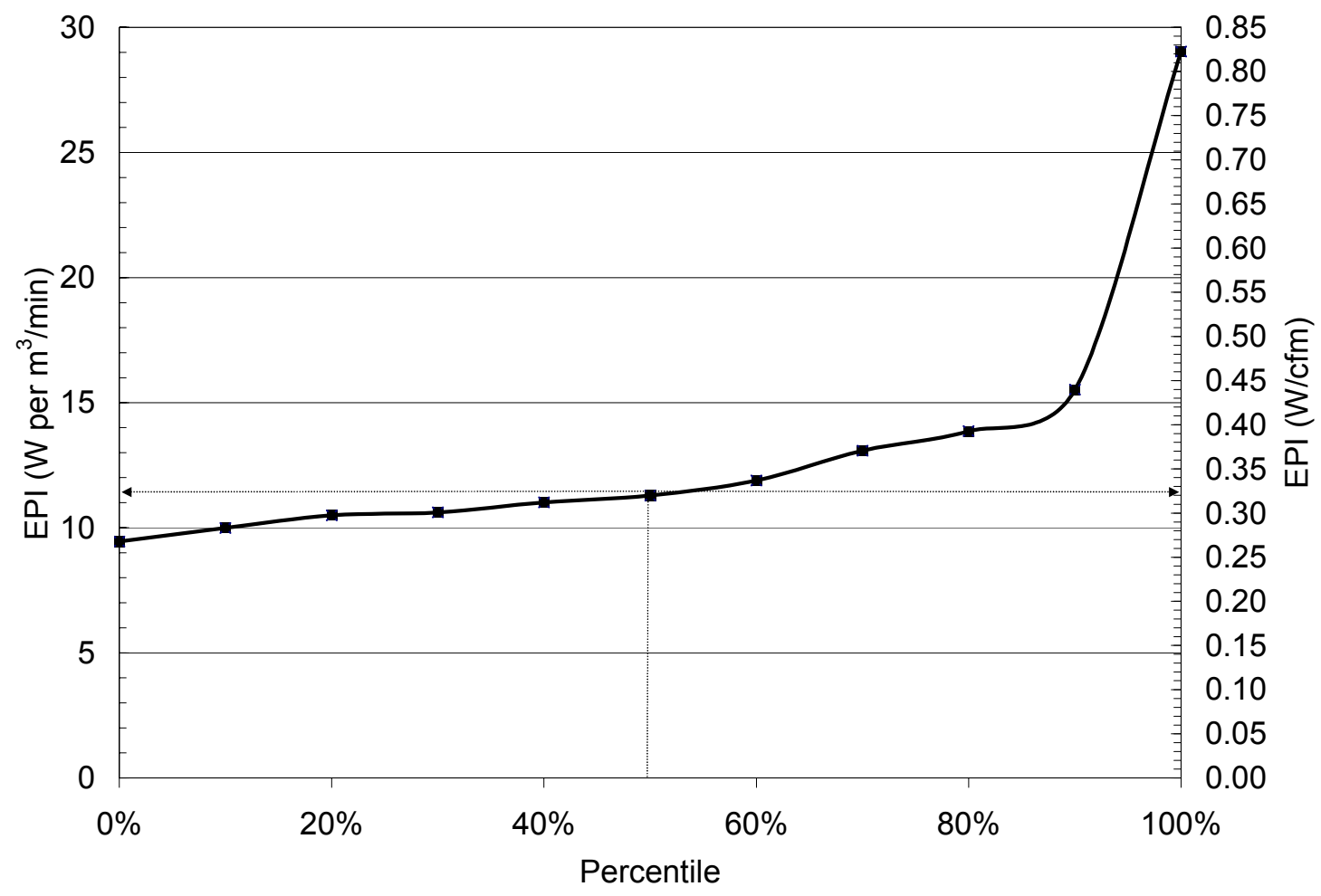

Figure 5 Distribution of FFUs' EPI (power intensity)

\section{Discussion}

When a method for consistently reporting the energy performance of fan-filter units is established and is adopted by the industry, FFU suppliers can then use it and report the 
performance data in a consistent manner. Cleanroom owners and designers can make informed selections of FFUs regarding energy usage and efficiency. The new laboratory method for energy performance being developed at LBNL requires experimental setup that is similar to the existing ITRI setup. While we do not intend to specify the minimum test-equipment requirements in terms of measurement accuracy and equipment calibration, which are normally part of relevant certification programs (e.g., AMCA fan certification), we recommend that users conduct FFU testing using equipment with sufficient accuracy that would be acceptable for good practice. It is hoped that the essence of this laboratory testing method can be integrated or adopted by a national organization such as IEST or ASHRAE in its developmental activities. IEST Working Group (WG) 36 is currently starting to develop a guideline on recommended practice for testing overall performance of FFUs, of which the scope is broader. The WG recommended practice probably will address in-situ testing and also include additional important parameters, such as noise and vibration performance.

We are expecting significant benefits of having such a method in place and used by the industry. For example, a success can be to provide comparable performance information to users and designers for them to make informed decisions such as selecting more energy efficient models. In addition, a market transformation could be accelerated through possible utility incentive programs based upon performance data consistently measured and analyzed using this method. Utilities and other public interest programs promoting energy efficiency may be able to encourage use of more energy efficient models. Furthermore, there can be a role that the IEST WG can play to carry forward this synergistic effort. In this regard, this study can be beneficial to the IEST WG, which may consider adopting essence of this method and recommendations into the new recommended practice that will be developed.

\section{Conclusions and Recommendations}

Laboratory testing of FFU energy performance can provide useful data for suppliers and end users to understand the performance of FFU products. The recommended energy metrics include the FFU Energy Performance Index (EPI) and total pressure efficiency. While the methods and results presented are intended to stimulate further investigations and may require additional industry input, the advantage of establishing such a consistent testing and evaluation method is to provide better understanding of FFU performance that can be comparable. New performance information produced in this manner can suggest good practices and energy-saving opportunities in FFU applications.

The outcomes presented in this paper are based upon a relatively small sample of new FFU products $(1220 \mathrm{~mm} \times 610 \mathrm{~mm}$, or $4 \mathrm{ft} \times 2 \mathrm{ft})$ in the market. While the evaluation method is developed and intended to compare FFU performance, it is premature to directly use results derived from this small sample size (i.e., median values of EPI and total pressure efficiency presented or discussed in this paper) for formulating a performance baseline. The following is recommended for future investigations:

- Evaluate FFUs' energy performance based upon additional criteria, such as static pressures setting at various levels (e.g., 100, $150 \mathrm{~Pa}$; or 0.4, 0.6 inch water), or face airflow speeds at certain levels (e.g., $0.35 \mathrm{~m} / \mathrm{s}$, or $70 \mathrm{fpm}$ ); 
- Investigate factors contributing to actual performance levels, such as motor types, fan wheels, design, and orientations of FFUs tested;

- Conduct tests of additional FFUs with various sizes and types; and

- Identify opportunities in design, operation, and control to improve FFUs' overall performance.

\section{Acknowledgements}

The project is funded by the California Energy Commission's Industrial section of the Public Interest Energy Research (PIER) program (http://www.energy.ca.gov/). This work was supported by the Assistant Secretary for Energy Efficiency and Renewable Energy, Office of Building Technology, State, and Community Programs, of the U.S. Department of Energy under Contract No. DE-AC03-76SF00098.

\section{References}

[1] Xu, T. 2004. Efficient Airflow Design for Cleanrooms Improves Business Bottom Lines. Journal of the IEST, Vol. 47 (2004 Edition), Institute of Environmental Sciences and Technology (IEST), Rolling Meadows, IL. An earlier version appeared in IEST Proceedings of The 49th Annual Technical Meeting: April 2003, Phoenix, AZ.

[2] Xu, T. 2003. Performance Evaluation of Cleanroom Environmental Systems. Journal of the IEST, Vol. 46: 66-73. Institute of Environmental Sciences and Technology (IEST), Rolling Meadows, IL.

[3] Lee, A.H, F. Karman. 2003. Energy Conservation Measures for Industrial Facilities. HPAC Engineering, July 2003: 45-49. 1300 E. 9th St. Cleveland, OH: www.HPAC.com.

[4] Jeng, M.S., F. Tsau. 2002. Fan-Filter Unit (FFU) Test Procedures. Semiconductor Fabtech, $15^{\text {th }}$ Edition: $77-80$.

[5] ASHRAE/ANSI Standard 51 (ANSI/AMCA 210-99). 1999. Laboratory Methods of Testing Fans for Rating, Air Movement and Control Association International, Inc., Arlington Height, IL, USA.

[6] Jeng, M. and W. Tschudi. 2002. Draft Standard Test Procedure For Fan-Filter Units (not published).

[7] Xu, T. (Ongoing.) Laboratory Method of Testing Energy Performance of Fan-Filter Units. Lawrence Berkeley National Laboratory Report, LBNL-54626, Berkeley, CA.

[8] International Organization for Standardization (ISO). 1999. ISO/DIS 14644-1 Cleanrooms and associated controlled environments. Part 1: Classification of Air Cleanliness. The Institute of Environmental Sciences and Technology (IEST), 5005 Newport Drive, Rolling Meadows, Illinois 60008-3841, USA. 
[9] International Organization for Standardization (ISO). 2002. ISO/DIS 14644-3: Cleanrooms and associated controlled environments. Part 3: Metrology and Test Methods. The Institute of Environmental Sciences and Technology (IEST), 5005 Newport Drive, Rolling Meadows, Illinois 60008-3841, USA.

[10] The Institute of Environmental Sciences and Technology (IEST). 1997. IEST-RP-CC006.2: Testing Cleanrooms. ISBN: 1-877862-26-6. 5005 Newport Drive, Rolling Meadows, Illinois 60008-3841, USA. 